\title{
Lamination microstructure in shear deformed copper single crystals
}

\author{
Olga Dmitrieva, Patrick W. Dondl, Stefan Müller, Dierk Raabe
}

November 10, 2008

\begin{abstract}
We investigate the formation of microscopic patterns in a copper single crystal deformed in a shear experiment. Using high-resolution electron backscatter diffraction (EBSD) imaging, we find a band-like microstructure consisting of confined areas in the sample with rotated lattice. Digital image correlation (DIC) allows us to exactly determine the macroscopic state of deformation of the sample. This data can be used as a side condition to calculate the lamination parameters from the theory of kinematically compatible lamination of separate material regions, each deforming in single slip. The parameters given by the theory agree with the measured properties, i.e., a lattice rotation of 3 degrees and a lamination normal rotated 7 degrees counterclockwise from a $\langle 111\rangle$ direction.
\end{abstract}

Keywords: plasticity; microstructure; laminates; single crystals; digital image correlation; EBSD

\section{Introduction}

Many studies on the behavior of metals under stress aim at understanding the evolution of plastic deformation. Since plastic behavior of materials is the result of the interaction of lattice defects at several length scales, the overall macroscopic properties are strongly influenced by the formation of internal microstructures. The main concern of this work is to obtain better understanding of deformation patterning phenomena in plasticity, particularly regarding the formation of laminates. These internal structures generally arise when the energy density of the body becomes non-convex. This leads to a frustration of the homogeneous state, which makes it energetically favorable for the material to develop a microstructure.

In plasticity such a loss of convexity in the energy can, for example, stem from the phenomenon of cross- (or latent-) hardening [4, 17, 12], when activity in one slip-plane suppresses activity in the others. The well known example of geometric softening, where the lattice in a crystal locally rotates until one slip system is oriented such that it can accommodate the applied load $[3,11,6,13]$ 
can be explained by a loss of convexity in the energy [19]. In fatigue experiments, as well as in experiments involving only a single-pass deformation, the formation of laminate-like microstructures has been observed $[22,15,5]$. This lamination behavior was explored by Ortiz and Repetto [19] who generally consider it a phenomenon stemming from cross-hardening.

In this work, we study the formation of lamination microstructure in plastically deformed copper single crystals under well-defined single-pass loading conditions. We measure the macroscopic deformation with the digital image correlation method and characterize the microstructure using electron backscatter diffraction. This allows us to quantitatively compare the predictions from the theory of laminates to the actually observed microstructure. To our knowledge, this is the first time of such a quantitative analysis of the microstructure formed during plastic deformation.

In simple shear experiments, nearly uniform macroscopic strain can be achieved with the use of a sample geometry in which friction effects are negligible in the region of interest $[14,26]$. The use of single crystals as specimens allows defined macroscopic activation of a subset of the twelve FCC slip-systems $\langle 110\rangle\{111\}$ (see Table 1) during deformation. The external plastic deformation is accommodated by mobile dislocations which carry the slip. The interactions between mobile and immobile dislocations and subsequent dislocation trapping lead to the hardening of the material. Regarding to the hardening effects, the plastic part of the stress-strain curve of single crystals is divided into several stages [10]. Stage I (easy glide) occurs only during single slip, the hardening rate is small compared to the other regions. Stage II (linear hardening) is characterized by a relatively high constant work hardening rate due to the dislocation interactions.

The load/displacement dependence during the shear deformation is measured by the mechanical loading device. The evolution and spatial distribution of the deformation is traced on the surface of the sample using digital image correlation (DIC). This allows to determine the macroscopic properties of the deformation. The microstructure of the sheared material was then characterized using orientation microscopy based on the analysis of the electron backscatter diffraction pattern (EBSD). In addition, a phenomenological crystal plasticity FEM-simulation was conducted, the macroscopic results of which qualitatively agree with the DIC-measurement.

In the present experiments we chose the orientation of copper single crystals considering the direction of the applied load such that two slip systems in a single slip plane have highest (and equal) Schmid factors. The DIC-analysis of the resulting strain shows a large region of almost homogeneous, affine deformation. The sample in this region is in simple shear, with the shear normal, however, slightly rotated with respect to the originally chosen slip plane. Therefore, a second set of slip systems must be activated to accommodate this macroscopic deformation. An analysis, assuming infinite cross-hardening (the material is in single slip at each material point) predicts a lamination microstructure - similar to the ones found in shape memory alloys - that corresponds almost exactly to the orientation patterning observed in the EBSD-measurement. 


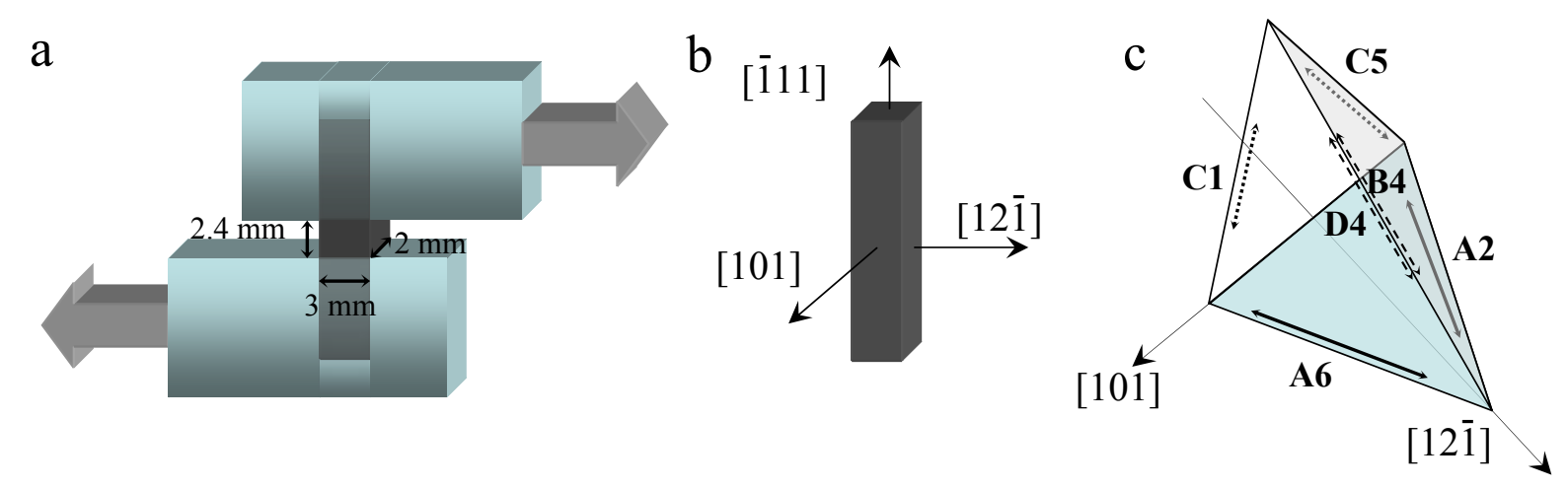

Figure 1: a: Schematic drawing of the specimen holder with the specimen used for the shear experiments; $\mathbf{b}$ : Crystallographic orientation of the single crystal sample positioned in the sample holder as shown in a:; c: Positions of the active slip planes according to the given crystallographic orientation, the individual slip directions are indicated by the arrows. The nomenclature is given in Table 1.

Slip systems in an FCC crystal

\begin{tabular}{ccccccccccccc}
\hline System & B2 & B4 & B5 & A3 & A2 & A6 & C1 & C3 & C5 & D4 & D1 & D6 \\
\hline$\sqrt{2} s$ & $\pm[0 \overline{1} 1]$ & $\pm[10 \overline{1}]$ & $\pm[\overline{1} 10]$ & $\pm[101]$ & $\pm[01 \overline{1}]$ & $\pm[\overline{1} \overline{1} 0]$ & $\pm[011]$ & $\pm[\overline{1} 0 \overline{1}]$ & $\pm[1 \overline{1} 0]$ & $\pm[\overline{1} 01]$ & $\pm[0 \overline{1} \overline{1}]$ & $\pm[110]$ \\
$\sqrt{3} m$ & $(111)$ & $(111)$ & $(111)$ & $(\overline{1} 11)$ & $(\overline{1} 11)$ & $(\overline{1} 11)$ & $(\overline{1} \overline{1} 1)$ & $(\overline{1} \overline{1} 1)$ & $(\overline{1} \overline{1} 1)$ & $(1 \overline{1} 1)$ & $(1 \overline{1} 1)$ & $(1 \overline{1} 1)$
\end{tabular}

Table 1: Schmid and Boa's FCC slip system nomenclature used in the text. For example, -B4 indicates a shear in the direction of $-[10 \overline{1}] \otimes[111]$. From [19].

\section{Experiments}

\subsection{Sample preparation and shear experiments}

The single crystal samples were cut from copper single crystal (99.98\%) produced by the melt-grow method. The specimens were cut by spark erosion, polished mechanically, and, subsequently, electrolytically. The dimensions of the single crystal samples are $3 \mathrm{~mm}$ by $2 \mathrm{~mm}$ with a height of $10-15 \mathrm{~mm}$. The shear tests were performed on a special miniaturized testing device by Kammrath \& Weiss GmbH (44141 Dortmund, Germany). The computerized device features two moveable crossheads allowing the sample to remain in a stable centered position during testing. The load was controlled by the load cell with maximal load value of $1 \mathrm{kN}$. The displacement with the deformation rate $5 \mu \mathrm{m} / \mathrm{sec}$ was measured by the machine extensometer. The position of the specimen in the sample holder of the testing device is schematically shown in Figure 1a. The distance between the sample holders, i.e., the length of the freestanding part of the specimen is $2.4 \mathrm{~mm}$.

Figure $1 \mathrm{~b}$ shows the initial orientation of the undeformed single crystal (101) $[12 \overline{1}]$. The deviation of the crystal lattice from the given orientation does 
not exceed 0.5 degrees. The shear load is applied along the $[12 \overline{1}]$ direction shown in Figure 1b. Under these loading conditions, two coplanar slip systems of the single crystal in the aforementioned crystallographic orientation have Schmid factors of magnitude approximately equal 0.63 (A2 and A6 in Table 1). These two slip systems are shown schematically in Figure 1c, the slip directions A2 and A6 are indicated by the arrows. The gray colored triangle corresponds to

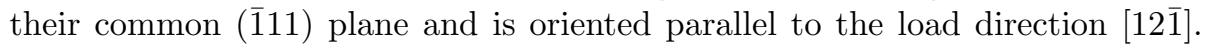
Since the primary active systems are coplanar (and thus their deformations are compatible), one can consider their sum as a single system for which the activation direction is the direction of the applied load. Other slip systems with the non-zero Schmid factors are also shown in Figure 1c in accordance with the nomenclature in Table 1.

\subsection{Digital image correlation method}

In order to measure the strain on the surface of the specimen in-situ, we applied the digital image correlation method (DIC) [21, 25]. The DIC method is based on the recognition of geometrical changes in the gray scale distribution of surface patterns after straining. In order to get a stochastic optical pattern, white acrylic spray was used for the background and graphite spray for optical decoration. For DIC we used Aramis System (version 6.0.0-3) by GOM Inc. equipped with two digital cameras (CCD-1300, maximal resolution 1280x1024 pixels) placed behind of the testing device. The recording time for each frame was set to 1 sec.

\subsection{Structural characterization using high resolution EBSD}

The structural characterization of the surface of the samples and the orientation microscopy were performed in the scanning electron microscope (SEM) Jeol JSM $6500 \mathrm{~F}$ with a field emission gun operated at $15 \mathrm{kV}$. The microscope is equipped with a detector for the imaging of backscattered electrons (BSE imaging). The electron back scatter diffraction pattern were recorded and evaluated by the EDAX/TSL EBSD System equipped with a Digiview camera. In the high resolution EBSD measurements the exposure time for each frame was set to about $0.5 \mathrm{~s}$ at the smallest binning size and for the calculation of the Hough transformation a binned pattern size $240 \times 240$ and an angular spacing control of 0.5 degrees were chosen.

\section{$2.4 \quad$ FEM}

The crystal-plasticity finite-element method (CP-FEM) has been applied for the simulation of the metal deformation [24]. A phenomenological crystal plasticity model based on the work of Kalidindi et. al. and Asaro [16, 1] was used in this study for the time integration of the constitutive equations. The elastoviscoplastic model was implemented in the finite element code MARC using the 


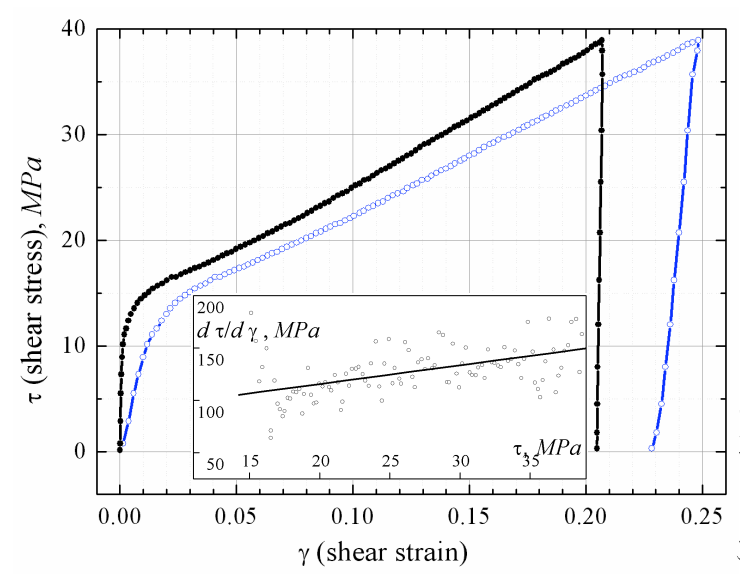

b

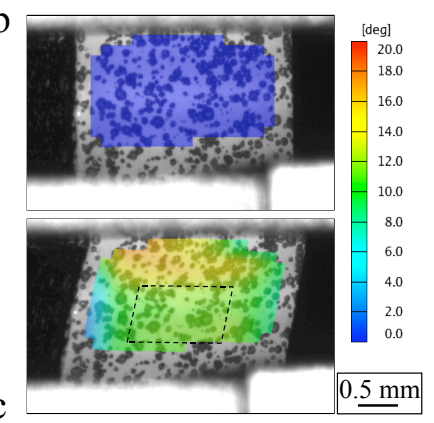

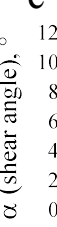

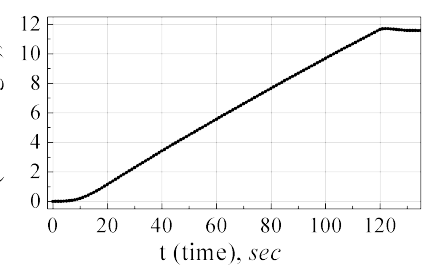

Figure 2: a: Shear stress $\tau /$ shear strain $\gamma$ dependence obtained from the load/displacement measurement (blue curve) and using the DIC method (black curve), respectively. Inset: The hardening rate $\mathrm{d} \tau / \mathrm{d} \gamma$ calculated for the plastic deformation part of the stress/strain curve estimated from the DIC. b: Digital images of the sample surface decorated for the DIC method before (upper image) and after the deformation (lower image) overlaid with the obtained distribution of the shear angles according to the color scale. c: Time resolved change of the shear angle during the deformation experiment estimated by the DIC method. The value of the shear angle was averaged over the central region of the investigated area as indicated in $\mathbf{b}$.

user-defined subroutine HYPELA2. We used a 3D finite element mesh with 2400 eight-node brick elements for the discretization of the sample.

\section{Experimental and FEM results}

\subsection{Deformation of the single crystal sample}

The single crystal specimens were deformed in simple shear. The shear strain of the plastic deformation as measured in the machine extensometer amounts to $\gamma=0.23$ (technical shear strain). The load/displacement dependence was recorded during the shear deformation with the step size of $5 \mu \mathrm{m}$. Figure $2 \mathrm{a}$ (blue curve) presents the dependence between the applied shear stress $\tau$ and shear strain $\gamma$ as calculated from the load/displacement data for the given sample geometry.

Above $\tau=15 \mathrm{MPa}$ the deformation of the specimen is predominantly plastic and the slope (the hardening rate $\mathrm{d} \tau / d \gamma$ ) of the approximately linear 


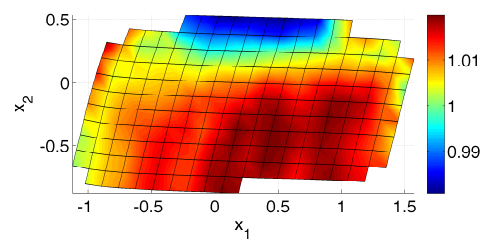

(a) $U_{11}$

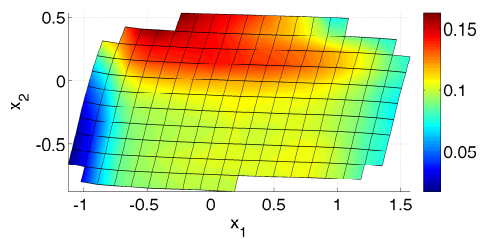

(b) $U_{12}$

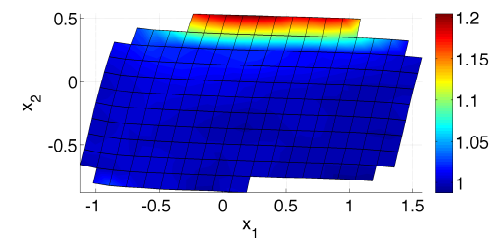

(c) $U_{22}$

Figure 3: The three 2d strain components from DIC-measurement

stress/strain dependence is nearly constant (inset in Figure 2a). The constant value of $\mathrm{d} \tau / d \gamma$ corresponds to stage II of the plastic deformation (linear hardening). In order to quantify the hardening rate during plastic deformation, we estimate the shear modulus $G$ from the slope of the unloading branch of the black curve to $G \approx 15 \mathrm{GPa}$. This value is somewhat lower than more exact the shear modulus obtained by measuring the ultrasonic velocities (20-80 GPa, depending on the crystal direction [20]), but lies in the same order of magnitude. We calculate the mean value of the hardening rate $\langle\mathrm{d} \tau / d \gamma\rangle=130 \mathrm{MPa}$ $\approx G / 120$. This value correlates to hardening rates in stage II (linear hardening) given in the literature for the deformation of single crystals $(\mathrm{G} / 200)$ [23, 18]. No occurrence of the stage I (easy glide) which is characterized by a single glide with a low hardening rate of about $G / 5000$ was observed here.

In the applied DIC approach, digital images of the specimen surface decorated with the optical pattern were recorded during the experiment. Figure $2 \mathrm{~b}$ shows the digital images of the sample before (upper image) and after the deformation (lower image). The presented images were overlaid with the obtained strain fields that reveal the distribution of the shear angles on the specimen surface in accordance to the color scale (right hand side on Figure 2b).

As seen in Figure 3, the DIC-measurement on the surface of the sheared copper single-crystal sample reveals an inhomogeneous deformation with some concentration of strain near the clamps. There is also a larger region of fairly homogeneous deformation in the central area of the sample.

The values of the shear angles of the homogenous deformed central region were averaged, and in Figure 2c time resolved change of the mean shear angle is shown. From this curve it follows that the residual plastic deformation amounts $\alpha=11.6^{\circ}(\gamma=0.20)$. Since the DIC images were recorded simultaneously with the load/displacement measurement, we get the dependence between the shear stress and shear strain estimated using DIC (black curve in Figure 2b). This curve gives the effective deformation behavior of the sample after excluding the contributions of the sample holder or test device.

The DIC strain measurement also reveals the distribution of macroscopic strain and of the deformation gradient on the surface of the deformed crystal. 


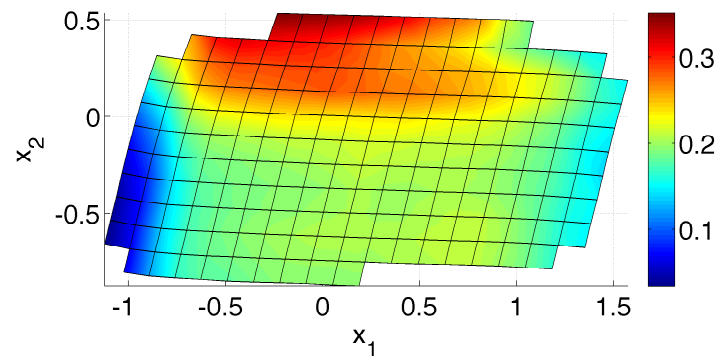

(a) Magnitude $\gamma$ of simple shear for the final deformation at $t=136 \mathrm{~s}$.

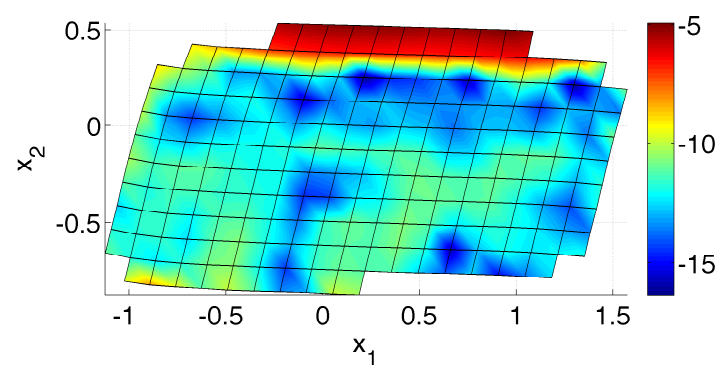

(c) Logarithm of the measure of difference between the strain $F$ and a simple shear $G$, $\ln (E(F, G))$ for the final deformation at $t=136 s$.

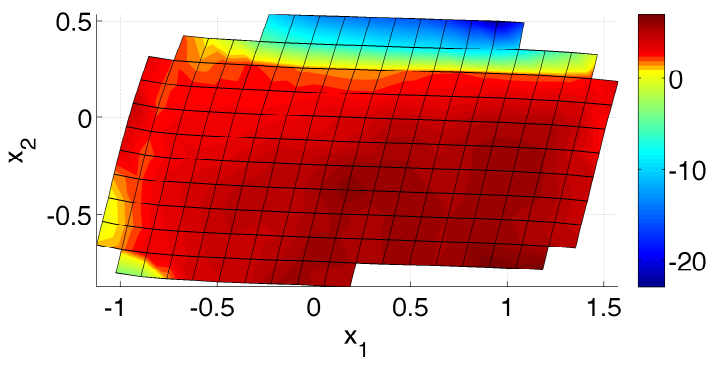

(b) Angle $\varphi$ of the shear normal, clockwise with respect to $x_{2}$ direction for the final deformation at $t=136 s$.

Figure 4: Shear magnitude, angle of the shear normal, and a measure of the distance of the DIC-measured deformation to the simple shear. 
In order to analyze the deformation in more detail, we compare the observed deformation gradient $F$ to a simple shear situation $G(\gamma, \varphi)$ with only two parameters: the magnitude $\gamma$ and the orientation of the shear normal with respect to the vertical (expressed by the angle $\varphi$ ). The comparison is done by minimizing an energy $E(F, G)$ that measures the difference between a given strain $G$ and the observed deformation gradient $F$. The energy used is akin to a frame indifferent elastic energy assuming that $F=F^{\mathrm{el}} G$, i.e., the deformation not covered by $G$ has to be accommodated by an elastic deformation $F^{\mathrm{el}}$. We choose

$$
E(F, G)=\left\|\sqrt{\left(F G^{-1}\right)^{\mathrm{T}}\left(F G^{-1}\right)}-\mathrm{Id}\right\|^{2} .
$$

Here, $\|A\|^{2}=\sum_{i, j} A_{i j}^{2}$ denotes the Hilbert-Schmidt norm of a matrix A. This Hilbert-Schmidt norm of a symmetric strain tensor is equivalent to linear elastic energy. The square root $T=\sqrt{A}$ of a positive semidefinite matrix is the unique positive semidefinite matrix such that $T^{2}=A$.

As one can see in Figure 4, the strain in the homogeneous region is very well approximated by a simple shear $G$ with a normal slightly - about $4-5$ degreesrotated clockwise from the vertical, since $E(F, G)$ is very small compared to $E(F, I d)$. Averaging the deformation gradient in the homogeneous region (rows $4-12$ and columns $3-19$ of the nodes in the DIC-figures, counting from top-left) yields

$$
\begin{gathered}
E\left(F_{\text {avg }}, G\right)=6.1 \cdot 10^{-6} \text { with } \\
G=\left(\begin{array}{c}
0.1991 \\
-0.0156
\end{array}\right) \otimes\left(\begin{array}{c}
0.0779 \\
0.9970
\end{array}\right)+\text { Id. }
\end{gathered}
$$

One can see that $G$ corresponds to a simple shear of magnitude $\gamma=0.1997$, and normal $n$ rotated clockwise by $\varphi=4.47^{\circ}$ from the vertical ${ }^{1}$.

To assess the overall deformation of the sample, the average deformation gradient over the whole sample as measured by DIC was calculated. Unfortunately, the DIC-matching software does not always recognize enough points in the deformed sample, especially in highly strained regions. Because of this, assuming symmetry, only the top half of the sample - up to measurement row 9 - could be used. This calculation reveals that the averaged total deformation $F_{\text {tot }}$ corresponds almost exactly to the intended boundary condition of a simple shear with vertical normal, so that

$$
E\left(F_{\text {tot }}, G\right)=1.5 \cdot 10^{-4} \quad \text { with } G=\left(\begin{array}{cc}
1 & 0.21 \\
0 & 1
\end{array}\right) .
$$

Given the imperfect pattern matching in DIC, that did not allow to consider particularly highly strained regions near the clamps, this shear of magnitude $\gamma \approx 0.21$ is in agreement with the value given by the machine extensometer. Figure 4(d) also shows that for an early stage of the deformation, at $t=30 \mathrm{~s}$,

\footnotetext{
${ }^{1}$ The shear parameters were obtained using a gradient flow-method to find the minimizers of $E(F, \cdot)$. It was implemented in MATLAB.
} 
a

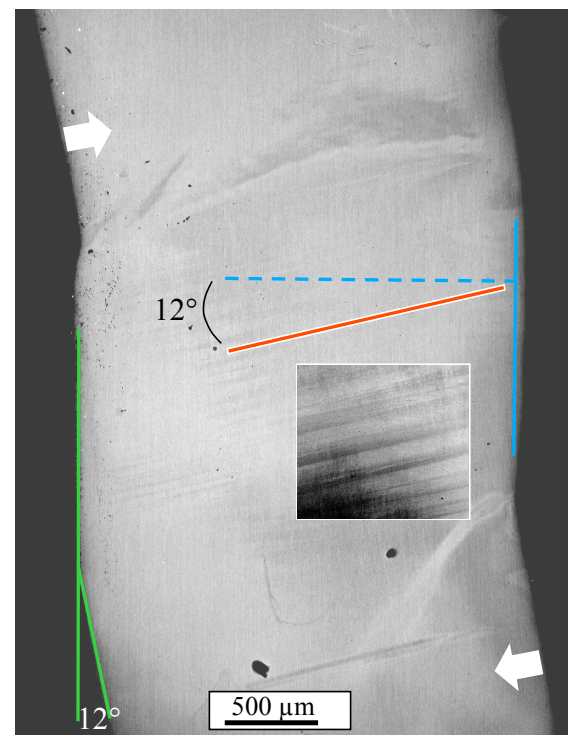

$\mathrm{b}$

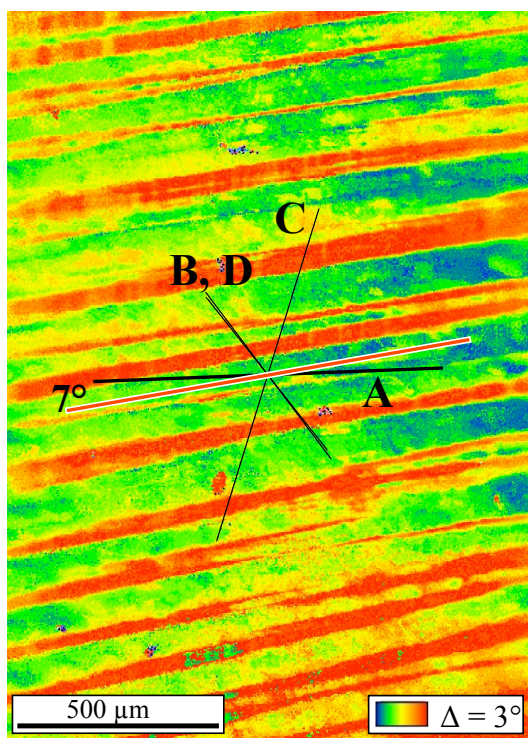

Figure 5: a: BSE overview micrograph of the copper single crystal after the shear deformation. The direction of the applied load is shown by the white arrows. With blue color the position of the side plane and its normal are drawn in. Inset: BSE micrograph of higher resolution placed adjusted to the scale. The angle between the microbands and the side plane of the deformed crystal amounts 12 degrees. b: EBSD map of area in the deformed part of the specimen which shows the relative crystallographic orientation changes within of 3 degrees according to the color scale. The plane traces of the slip planes are presented as black lines and labeled according to the nomenclature given in Table 1. The direction of the microbands which are tilted to 7 degrees with respect to the primary slip plane $A$ is signified by red line.

the bulk part of the sample already shows a variation of the shear normal away from the glide plane of the primary slip system. This explains why we experience stage II plasticity starting early in the deformation process, since more than one glide plane has to be active to accommodate this deformation.

\subsection{Structural characterization of the deformed sample}

\subsubsection{BSE overview imaging}

Figure 5a shows the BSE micrograph of the single crystal after shear deformation. The direction of the applied shear load is indicated by white arrows. From the geometry of the deformed sample one can estimate that the freestanding side plane of the crystal is tilted after the shear deformation to about 12 degrees as marked with the green lines. With the blue lines on the right hand 
side we marked again the position of the side face and its normal direction. In this micrograph, the formation of glide bands can be observed in the deformed part of the crystal. In the inset of Figure 5a we present a BSE-micrograph of higher resolution which provides more details and a higher BSE contrast. The direction of the bands is than indicated by the red line. The angle between the observed bands and the side plane of the deformed part amounts than about 12 degrees. Taking into account that the tilt of the side plane was also 12 degrees, one can conclude that the microbands of the deformed single crystal are oriented parallel to the initial position of the crystallographic plane of the primary slip, i.e., are aligned along the direction of the applied shear load. The areas with the observed glide bands were characterized using EBSD analysis.

\subsubsection{EBSD characterization}

Figure 5b shows an EBSD map of the deformed part of the crystal. The measurement was done with the scanning step size of $5 \mu \mathrm{m}$. The EBSD map shows the relative variations of the orientation of the crystal lattice within 3 degrees according to the color scale. The reference orientation relates to the blue signed areas. The formation of the microbands with a different orientation (red) compared to the material in between (green/blue) can be observed from this map. Using the evaluation software from TSL, the plane traces of the $\{111\}$ slip planes are displayed according to the averaged lattice orientation of the investigated area as shown in Figure 5b. As shown previously in Figure 1c, the common slip plane of the primary active slip systems is initially oriented parallel to the shear load (along $\langle 112\rangle$ ). This plane trace is drawn in Figure 5b by the thick black line and marked as $A$. When comparing the direction of this $\{111\}$ plane with the direction of the microbands (displayed as a red line), a deviation of about 7 degrees can be determined. Thus, the direction of the microbands is not crystallographic. Furthermore, since the development of the microbands occurs along the direction of the applied load and parallel to the initial position of the primary slip plane, a macroscopic tilt of the crystal lattice out of the initial state to about 7 degrees can be determined in the final state of the deformation.

Figure 6a shows an EBSD map of part of the deformed area containing some microbands. The measurement was done using a scanning step size of $2 \mu \mathrm{m}$. From this micrograph, a further feature of the bands can be observed, namely the additional patterning inside of the "red" microbands which are subdivided into parts by orthogonal intersections (ladder-like substructure).

\subsubsection{High resolution EBSD}

Figure $6 \mathrm{~b}$ shows the high resolved EBSD measurement of the area that includes the microband and the surrounding material. The step size of the scanning measurement was $0.1 \mu \mathrm{m}$. The EBSD map presents the orientation distribution relative to a reference point (initial orientation) according to the color scale within of 3 degrees. The red-colored zones in Figure $6 \mathrm{~b}$ shows the area on the edge of the microband. The green/blue areas correspond to the material outside 

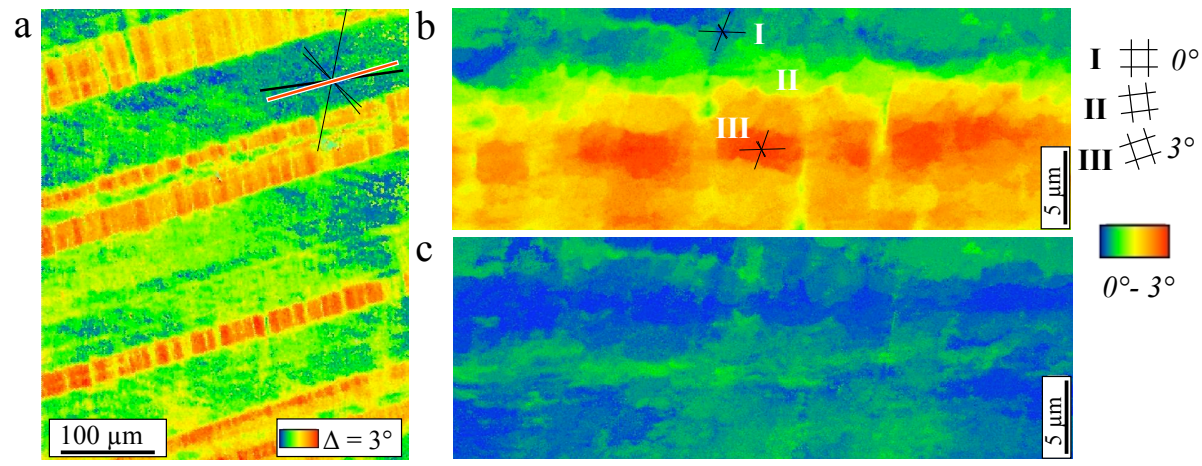

Figure 6: a: EBSD map of the part of the deformed area with several microbands. The map shows the relative crystallographic orientation changes within of 3 degrees according to the color scale. The plane traces of the slip planes are presented in the right corner.b: High resolution EBSD map on the edge of a microband (lower part). The colors indicate the distribution of the crystal orientation relative to the reference point (initial orientation) within 3 degrees. The plane traces of the slip planes are presented for the areas inside (red) and outside (green/blue) of the microband. Graphical illustration of the observed local lattice rotation is presented on the right hand side. c: High resolution EBSD map on the same area shows variation of the crystal orientation out of the normal direction according to the color scale. 


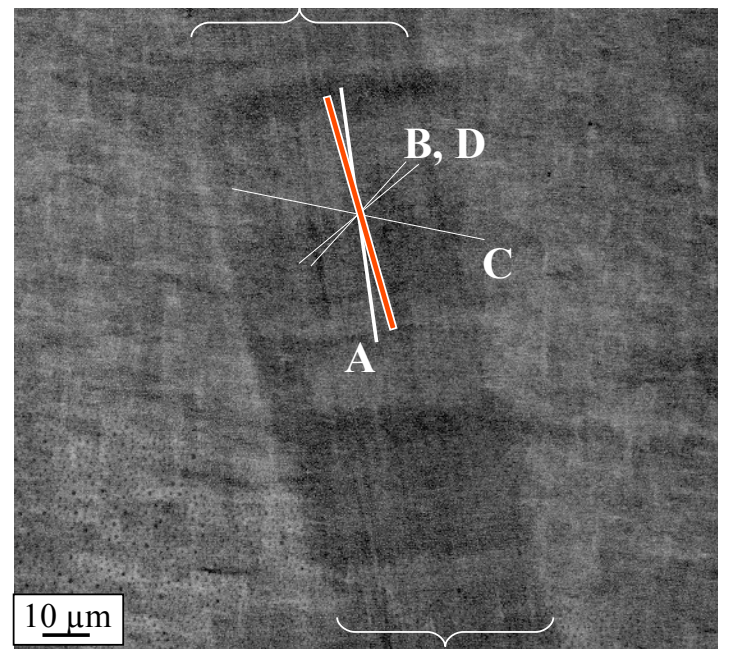

Figure 7: The SEM micrograph produced by the BSE imaging which shows a single microband (marked with braces). The positions of the slip plane traces in comparison to the direction of the microband (red line) are shown with white lines.

of the microband. The orientation difference indicates that the crystal lattice inside of the band is titled to 3 degrees in comparison to the material outside of the band.

In order to understand in which way the material rotates, we used an alternative representation of the EBSD results (Figure 6c). In Figure $6 \mathrm{c}$ the variation of the crystal orientation out of the normal direction is displayed in the same orientation deviation range as in Figure $6 \mathrm{~b}$. Figure $6 \mathrm{c}$ reveals no significant rotation of the lattice out of the front face. This observation shows that the lattice rotation of approximately 3 degrees occurs within the plane. In Figure $6 \mathrm{~b}$ the positions of the plane traces for measurement points inside and outside of the band are displayed. As schematically shown on the right hand side in Figure 6b, the rotation of the lattice inside of the band occurs counterclockwise compared to the outside region.

\subsubsection{BSE imaging of the microbands}

The microstructure of the deformed single crystal was investigated using BSE imaging. The BSE image in Figure 7 presents an area of the deformed part of the crystal which contains a single microband. The position of the microband is indicated by braces.

We discuss which information can be extracted from the BSE contrasts observed for the deformed specimen (Figure 7). The electrolytic polishing applied additionally after the deformation leads to a negligible topographic roughness of the crystal surface. Therefore, the topographic- and also the material contrast 
can be neglected here. One can assume that the structures observed in the BSE images correspond to pure electron channeling contrast. The electron channeling contrast arises due to the dependence of the intensity of the backscattered electrons on the fulfillment of the Bragg condition, i.e., the intensity depends on the tilt angle of the lattice with respect to the incident beam. For a small local tilt of the lattice, the contrast is induced predominantly by the superposition of strain fields from dislocations in the body [9, 27]. As already shown, no significant tilt of the lattice with respect to the incident beam (i.e., no tilt out of the plane of the investigated surface) both in the microband and in the surrounding material is observed. Therefore, we assume that the observed contrast in the BSE images arises predominantly from strain fields of trapped dislocations in the deformed single crystal. Inside of the microband in Figure 7 the bright lines aligned with the trace of the slip plane $A$ can be observed. Thus, some strain fields produced by the lattice distortion due to the storage of dislocations can be assumed along the slip plane $A$. This could be associated with the trapping of the mobile dislocation inside of the slip plane due to interactions between the dislocations of the slip systems A2 and A6 inside the slip plane $A$ or due to the intersection of this mobile dislocations with some forest dislocations. The enhanced mobility of the dislocations in the slip plane $A$ suggests activation of the corresponding slip systems A2 and A6 inside the microbands.

\subsection{Crystal plasticity finite element simulation of the shear experiment}

The deformation found in the crystal plasticity simulation described in section 2.4 qualitatively agrees with the digital image correlation measurement. We see a large inhomogeneous deformation near the fixed boundary on the top and the bottom of the sample. In particular, the deformation is high near the corners. There is also a large, homogeneously deformed region in the center of the sample.

In this central region, the deformation amounts to a simple shear, with a shearing normal rotated slightly clockwise with respect to the [111] crystal axis of the sample. The rotation is only approximately 1 degree, as opposed to the 4.5 degree rotation found in the DIC measurement. Still, qualitatively, this

would result in the same kind of microstructure as calculated in the following section.

\section{Energy minimizing microstructure}

In the following we introduce the theoretical framework in which we use to analyze the experimentally observed dislocation microstructure described above. We rely on a continuum finite plasticity model, assuming strong cross-hardening, as described by Ortiz and Repetto [19]. 


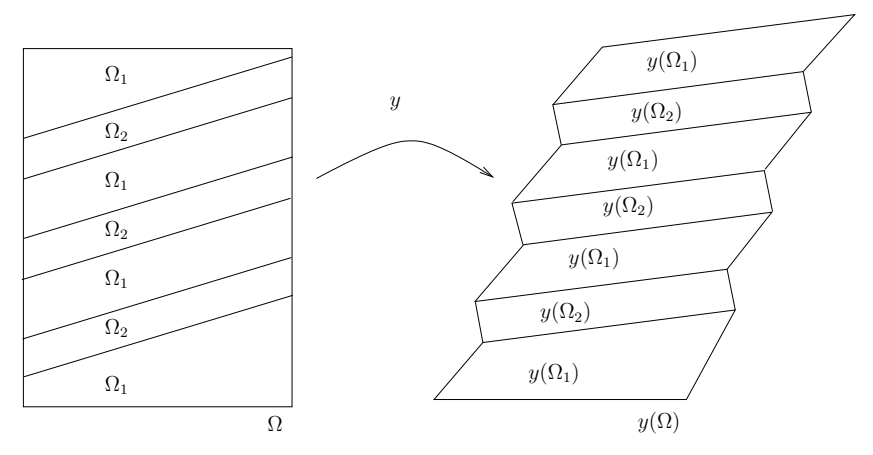

Figure 8: Reference and deformed configuration. A piecewise affine deformation is sketched, where $\nabla y(x)$ equals one constant tensor for $x \in \Omega_{1} \subset \Omega$ and a different constant tensor for $x \in \Omega_{2} \subset \Omega$.

\subsection{Loss of convexity and formation of microstructure}

Consider, using the framework of continuum mechanics, a body in a reference configuration $\Omega \subset \mathbb{R}^{3}$ and a smooth enough deformation $y: \Omega \rightarrow \mathbb{R}^{3}$, as illustrated in Figure 8. The deformation gradient is denoted by $\nabla y=F$. To illustrate the key ideas we now consider the extreme case of an ideally plastic, elastically rigid, solid with infinite cross-hardening. This corresponds to an energy functional for the crystal of the form

$$
E(F)= \begin{cases}0 & \text { if } Q F=\gamma s_{i} \otimes m_{i}+\operatorname{Id} \text { for } i \in\{1, \ldots, 12\} \text { and } Q \in S O(3) \\ \infty & \text { otherwise. }\end{cases}
$$

In other words, the energy density is zero, as long as one can find a slip system $s_{i} \otimes m_{i}$ accommodating the deformation gradient up to a rigid rotation $Q$. In between these rays of single slip deformation the energy is large (in fact, it is infinite), which renders the function non-convex. As we will see in the next section, this leads to the phenomenon that through the formation of a fine structure, for example by alternating layers of material respectively deformed in different single slip, one can accommodate, in an average sense, a larger variety of deformation gradients besides the obvious macroscopic single slip. The important restriction, however, is that the whole deformation has to be kinematically compatible, i.e., $F$ has to be the gradient of a continuous function.

\subsection{Piecewise affine deformations}

A frequently observed type of kinematically compatible microstructure consists of so-called rank-one laminates. Assume that the deformation is piecewise affine and that there are only two affine components, i.e., $\nabla y=F_{1}$ in $\Omega_{1} \subset \Omega$ and $\nabla y=F_{2}$ in $\Omega_{2}=\Omega \backslash \Omega_{1}, F_{1}$ and $F_{2}$ constant (as illustrated in Figure 8). This means that the deformation gradient is a certain constant $F_{1}$ in a subset of the reference configuration denoted by $\Omega_{1}$, and a different constant $F_{2}$ in the rest of the body denoted by $\Omega_{2}=\Omega \backslash \Omega_{1}\left(\Omega\right.$ without $\left.\Omega_{1}\right)$. 


\begin{tabular}{|c|c|c|c|c|c|c|c|}
\hline & $\begin{array}{r}\text { Choice of } \\
\text { slip systems }\end{array}$ & $\lambda$ & $\gamma_{P 1}$ & $\gamma_{P 2}$ & $\alpha$ & $\beta$ & $E$ \\
\hline & $P_{2}=\mathrm{B} 4+\mathrm{D} 4$ & $2 / 3$ & 0.35 & 0.042 & $-0.19^{\circ}$ & $-1.9^{\circ}$ & $5.0 \cdot 10^{-6}$ \\
\hline$\rightarrow$ & $P_{2}=\mathrm{B} 4+\mathrm{D} 4$ & $1 / 3$ & 0.18 & 0.082 & $-7.5^{\circ}$ & $3.8^{\circ}$ & $5.0 \cdot 10^{-6}$ \\
\hline$\rightarrow$ & $P_{2}=\mathrm{C} 1-\mathrm{C} 5$ & $2 / 3$ & 0.28 & -0.040 & $-6.3^{\circ}$ & $3.3^{\circ}$ & $5.0 \cdot 10^{-6}$ \\
\hline & $P_{2}=\mathrm{C} 1-\mathrm{C} 5$ & $1 / 3$ & 0.14 & -0.074 & $-14.3^{\circ}$ & $-5.6^{\circ}$ & $5.0 \cdot 10^{-6}$ \\
\hline
\end{tabular}

Table 2: Results for matching the strain $U$ from (6) with a lamination of slip systems. The value $\lambda$ is the volume fraction of the area deformed in slip $P_{2}$. The values $\gamma_{i}$ signify the magnitude and direction of slip in the $i$ slip-component, $\alpha$ is the angle between the [111]-direction in the area of larger volume fraction and the lamination normal, measured clockwise. The value $\beta$ is the angle of lattice rotation (clockwise) of the region of smaller volume fraction. $E$ is the remaining energy from the gradient flow. All rotations are exactly in the [101] plane. In all cases, $P_{1}=\mathrm{A} 2-\mathrm{A} 6$ (this denotes an equally large activity of the A2 slip system with positive sign from Table 1 and the A6 system with negative sign). Also, of the two solutions of equation (5), the one with twinning normal closer to the [111]-direction was picked, since the other solution would give an entirely different picture.

Under this condition, the requirement of kinematic compatibility is equivalent to the existence of an invariant plane, i.e., a plane that is stretched equally by both $F_{1}$ and $F_{2}$. The two differently deformed material domains can then be "glued together" on this invariant plane, as illustrated in Figure 8. This relationship can be expressed by requiring that

$$
Q F_{1}-F_{2}=b \otimes n,
$$

where $Q$ is a rotation matrix, $n$ is a normal vector and $b$ is a vector. This is also known as the Hadamard jump condition. The two deformation gradients are then compatible along an invariant plane with normal $n$. One can show that, for $F_{1} \neq F_{2}$, this equation either has no solution or it has two solutions [2].

This structure is very important in the theory of the martensitic phase transformation, where equation (5) is known as the twinning equation. For more information, refer to [7]. We will use the twinning equation to connect two regions with activity in different slip-systems to form an energy minimizing microstructure, assuming strong cross-hardening.

\subsection{A lamination microstructure accommodating the mea- sured macroscopic boundary values}

We consider the orientation of the sample during the shear experiment as illustrated in Figure 1. It is clear that a completely homogeneous shear of this type can be accommodated by an activity in the A2 and A6 slip systems, since $\gamma \cdot[12 \overline{1}] \otimes[\overline{1} 11]=\gamma \cdot([01 \overline{1}] \otimes[\overline{1} 11]-[\overline{1} \overline{1} 0] \otimes[\overline{1} 11])$. These slip systems are naturally compatible along the $[\overline{1} 11]$ direction. Due to the free boundary conditions, 


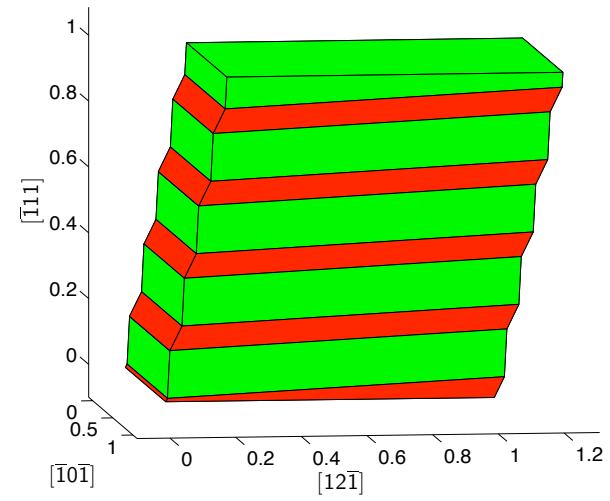

(a) A2-A6 activity in the small volume fraction "red" region. The secondary slip system $\mathrm{C} 1-\mathrm{C} 5$ is active in the green colored area.

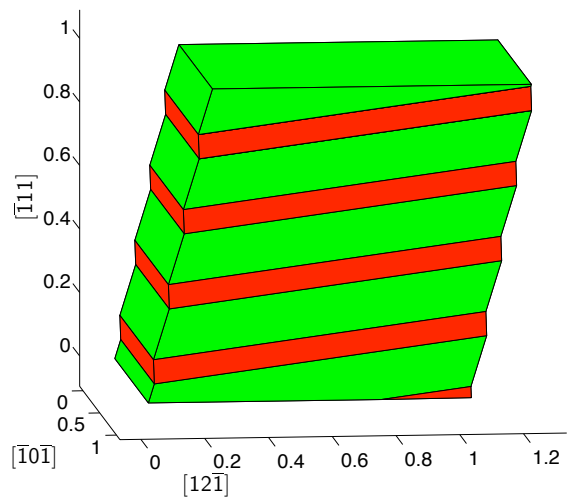

(b) B4+D4 activity in the small volume fraction "red" region. The primary slip system A2-A 6 is active in the green colored area.

Figure 9: Illustration of the two laminates that agree well with the measured macroscopic strain. One can see that both of them average to approximately the same deformation. The green and red areas correspond to the respective areas in Figure 5.

there is therefore a variety of possibilities to construct this deformation as a kinematically compatible plastic deformation.

The DIC-measurement, however, reveals that the actual deformation differs somewhat from this simplified assumption. Instead, there is a large, fairly homogeneous region (taken as above in Section 3.1) with average strain

$$
U=\left(\begin{array}{ccc}
0.9555 & -0.0198 & 0.0445 \\
& 1.1036 & 0.0198 \\
& & 0.9555
\end{array}\right)
$$

in the final deformation state. In calculating this average strain by changing the basis from the one in the DIC-measurement to the crystal directions, we assume that the deformation normal to the crystal surface is the identity.

A MATLAB program was employed to find a deformation, satisfying (5), with the two deformation gradients being simple shear in FCC slip systems, which - on average - yields the macroscopic strain (6) from the measurement. The program uses a gradient flow method for the magnitudes of slip in the involved slip systems with respect to the energy

$$
E=\left\|\sqrt{\left(U P^{-1}\right)^{\mathrm{T}} \cdot\left(U P^{-1}\right)}-\mathrm{Id}\right\|^{2}
$$

where $P=(1-\lambda) Q\left(\gamma_{1} P_{1}+\mathrm{Id}\right)+\lambda\left(\gamma_{2} P_{2}+\mathrm{Id}\right)$. Here, $\lambda$ is the volume fraction of one part of the laminate, $P_{1}$ and $P_{2}$ are the two displacement gradients occurring 
in the laminate, and $Q$ is the lattice rotation from the twinning equation (5) under which they are compatible. Equation (7) is therefore a relaxation of the energy in (4) that allows for a small elastic deformation.

The major plastic deformation component is an equal (opposite sign) activity in the aforementioned A2 and A6 slip systems, which are naturally kinematically compatible among each other without a rotation $Q$. One can therefore consider this slip as one part of the laminate.

For the secondary component of plastic deformation, there are of course several different choices that would be compatible with this deformation. Two of those choices are particularly natural, since they are compatible regardless of the magnitude of slip: First, an equal mixture of C1 and C5 (opposite sign), second, an equal mixture of B4 and D4 (same sign). Geometrically, the three slip system combinations A2-A6, C1-C5, and B4+D4 (nomenclature from Table 1) form a closed loop on the tetrahedron in Figure 1c.

One also has the choice as to which volume fraction is attributed to which slip. Using the histogram of the distribution of lattice rotations on the whole homogeneously deformed part of the face of the crystal, it can be determined that the "green" parts of Figure 5 occupy approximately two thirds of the total area in the picture. This area can now either be the primary component in the plastic deformation or the secondary component.

The results of the MATLAB gradient flow program are displayed in Table 2. It can be clearly seen that the $\mathrm{A} 2-\mathrm{A} 6$ and $\mathrm{C} 1-\mathrm{C} 5$ combination as well as the $\mathrm{A} 2-\mathrm{A} 6$ and $\mathrm{B} 4+\mathrm{D} 4$ combination yields lamination parameters almost exactly as observed in the experiment (marked by an arrow in the table), with the large volume fraction attributed to secondary and primary slip, respectively. The resulting deformations are illustrated in Figure 9.

This means first that the calculated lamination direction is rotated approximately 7 degrees counterclockwise with respect to the [111] direction in the region with larger volume fraction. Second, the lattice in the area of smaller volume fraction (red regions in Figures 5c and 6a) is rotated counterclockwise by approximately 3 degrees in comparison to the neighboring area.

The BSE imaging discussed in paragraph 3.2.4 leads to the assumption that inside of the "red" microbands the shear activity of A2 and A6 slip systems dominates. This would imply the choice of the combination A2-A6 for primary slip inside of the microbands and $\mathrm{C} 1-\mathrm{C} 5$ as the secondary active slip in the areas outside of the microbands.

It should also be noted that the assumption of infinite cross-hardening leads to slightly larger values for both the lamination angle and the lattice rotation in the calculation. The difference between the measurement and the calculation could therefore be explained by a small amount of mixing of the two slip systems.

Furthermore, the appearance of the substructure in the microbands which is observed in the orientation map in Figure 6a can be attributed to the presence of a small amount of different slip systems activated inside of the microbands. Their interaction can lead to the observed inhomogeneities in the slip distribution.

Of course, it is important that this microstructure is accessible though the 


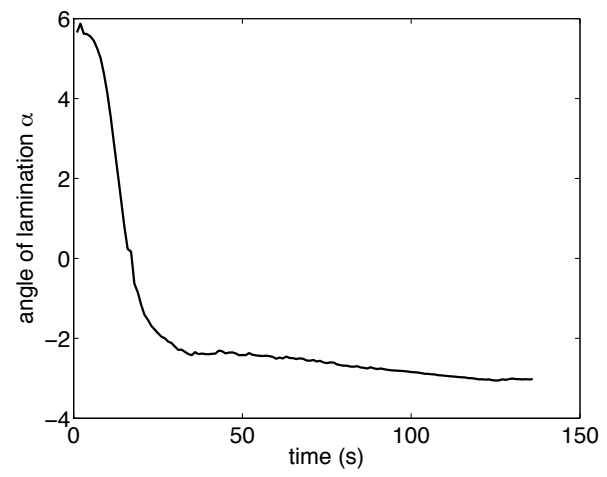

Figure 10: Change of lamination angle (in the reference configuration) for $P_{2}=$ C1-C5 during the course of the plastic deformation

dynamic process of the shear experiment, i.e., that there is a continuous path from an undeformed body to the final microstructure. For this, it is necessary that the lamination angle (denoted $\alpha$ in Table 2) does not change significantly during the deformation. Because the DIC-data is available for the intermediate time-steps during the deformation, this angle can be calculated for the whole process of the deformation using equation (5) in the same way as it was done for Table 2. In Figure 10, the evolution of this angle $\alpha$ is shown. One can see that, after an initial phase, where the deformation is very small anyhow, $\alpha$ is nearly constant. The calculation of this angle is done in the reference configuration (as opposed to the values in Table 2, which are calculated in the final configuration), in order to disregard that change in lamination angle due to the shearing of the lamination normal itself. The other parameters, like $\gamma_{i}$, evolve almost linearly over the course of the shearing. This means that despite increasing activity in the slip systems, the lamination structure itself can remain unchanged.

In summary, we have seen that a theoretical analysis assuming infinite crosshardening can predict a lamination microstructure in the shear-deformed copper single crystal that corresponds almost exactly to the orientation patterning observed in the EBSD-measurement.

\section{Conclusions}

In the present experiments we performed shear deformation on copper single crystals oriented such that two slip systems are primarily active. DIC-analysis of the resulting strain shows a large region of almost homogeneous deformation. The sample in this region is in simple shear, with the shear normal slightly rotated with respect to the originally chosen slip plane normal. Therefore, a second set of slip systems had to be activated to accommodate this macroscopic deformation, which can lead to the formation of a microstructure in the deformed single crystals. In high-resolution EBSD measurements we found mi- 
crobands that consist of regions where the crystal lattice is rotated by 3 degrees. The bands are aligned on a plane with normal rotated approximately 7 degrees away from a [111] direction in the crystal.

In this article, we showed that the theory of kinematically compatible lamination can predict such a microstructure. The basic assumption is that of infinite cross-hardening, which is satisfied fairly well in an fcc-crystal with low stacking fault energy like copper. This cross hardening leads to the fact that each material point can only deform in single slip. These regions of single slip then have to satisfy the Hadamard jump-condition, in order to avoid long-range elastic forces (and therefore a high elastic energy), and the average of the deformation gradients has to coincide with the macroscopic strain.

In our case, we were able to measure the macroscopic strain using the method of digital image correlation. This, together with some assumptions on the active slip systems, is enough to calculate the parameters of the formed microstructure. We find very good agreement of the lattice rotation and the lamination normal between the theoretical prediction and the EBSD measurement.

The finite element calculation that was performed yields a similar macroscopic deformation. The slip systems activated there, however, are impossible to arrange in a kinematically compatible fashion. Of course, the FEM-simulation can not, due to insufficient resolution, predict a lamination microstructure. Therefore, the simulation has to ignore compatibility and pay the higher energy required for the activation of more than one slip system at the same pointdespite cross-hardening - which explains the indifference of the simulation towards compatibility conditions. Including certain kinds of lamination could be a way to improve FEM-simulations of crystal plasticity.

We conclude that in this experiment, a lamination-type microstructure was observed. The question whether such a microstructure can be found under general loading conditions in finite plasticity is an open mathematical problem. For linearized crystal plasticity, this problem has been solved with a positive answer [8]. A further open question is that regarding the dynamics of such deformation, i.e., whether or not a certain microstructure can be formed given the whole time-dependent process of deformation. We assume that the lamination normal can not change significantly over time, since this would automatically contradict the assumption of strong cross-hardening. The general problem of the dynamics of pattern formation in single crystal plasticity is currently being investigated.

\section{Acknowledgments}

We are grateful to Dr. E. Hieckmann from Technische Universität Dresden for the helpful discussions concerning BSE analysis and to Dr. F. Roters from MaxPlanck-Institute for Iron Research for help with the FEM simulations. This work was supported by Deutsche Forschungsgemeinschaft (DFG Forschergruppe 797) and by the Triple M project of the Max Planck Society. 


\section{References}

[1] R. J. Asaro. Adv. Appl. Mech., 23:1-115, 1983.

[2] J. M. Ball and R. D. James. Fine phase mixtures as minimizers of energy. Arch. Rational Mech. Anal., 100(1):13-52, 1987.

[3] C.S. Barrett and L.H. Levenson. Trans. Metall. Soc. AIME, 137(112), 1940.

[4] J.L. Bassani and T.Y. Wu. Proc. Roy. Soc. London. Ser. A., 435(2141), 1991.

[5] B. Bay, N. Hansen, D. A. Hughes, and D. Kuhlmann-Wilsdorf. Evolution of f.c.c. deformation structures in polyslip. Acta Metallurgica et Materialia, 40(2):205-219, 1992.

[6] A.J. Beaudoin, H. Mecking, and U.F. Kocks. Phil. Mag. A, 73(1503), 1996.

[7] K. Bhattacharya. Microstructure of Martensite: Why It Forms and How It Gives Rise to the Shape-Memory Effect. Oxford University Press, 2003.

[8] Sergio Conti and Michael Ortiz. Dislocation Microstructures and the Effective Behavior of Single Crystals. Arch. Rat. Mech. Anal., 176(1):103-147, 2005.

[9] M.A. Crimp. Microscopy Research Technique, 69(374), 2006.

[10] J. Diehl. Z. Metall., 47331, 1956.

[11] I.L. Dillamore and H. Katoh. Met. Sci., 13(73), 1974.

[12] P. Franciosi and A. Zaoui. Acta Metallurgica, 30(1637), 1982.

[13] J.-H. Han, D.-I. Kim, K.-K. Jee, and K.H. Oh. Mat. Sci. Engng., 387(60), 2004.

[14] M.F. Horstemeyer, M.I. Baskes, and S. J. Plimpton. Acta Mater., 49(4363), 2001.

[15] N.Y. Jin and T. Winter. Cyclic deformation of copper single crystals oriented for double slip. Acta Metallurgica, 32:989-995, 1984.

[16] S. R. Kalidindi, C. A. Bronkhorst, and L. Anand. J. Mech. Phys. Solids, 40:537-569, 1992.

[17] U.F. Kocks. Trans. Metall. Soc. AIME, 230(1160), 1964.

[18] F.R.N. Nabarro, Z.S. Basinski, and D.B. Holt. Adv. Phys, 13(193), 1964.

[19] M. Ortiz and E. A. Repetto. Nonconvex energy minimization and dislocation structures in ductile single crystals. J. Mech. Phys. Solids, 47(2):397462, 1999. 
[20] W. C. Overton and J. Gaffney. Phys. Rev, 98(4):969ff, 1956.

[21] D. Raabe, M. Sachtleber, Z. Zhao, F. Roters, and S. Zaefferer. Acta Mater., 49(3433), 2001.

[22] K Rasmussen and O. Pedersen. Fatigue of copper polycrystals at low plastic strain amplitudes. Acta Metallurgica, 28:1467-1478, 1980.

[23] A.D. Rollett and U.F. Kocks. Solid State Phenomena, 35-36(1-18), 1993.

[24] F. Roters. Comp. Mat. Sci, 32:509-517, 2005.

[25] M. Sachtleber, Z. Zhao, and D. Raabe. Materials Science and Engineering, 336(81), 2002.

[26] P. Tugsu, P.D. Wu, and K. W. Neale. Intern. J. Plast., 18(1219), 2002.

[27] A.J. Wilkinson, G.R. Anstis, J.T. Czernuszka, N.J. Long, and P.B. Hirsch. Phil. Mag. A, 68(59), 1993. 